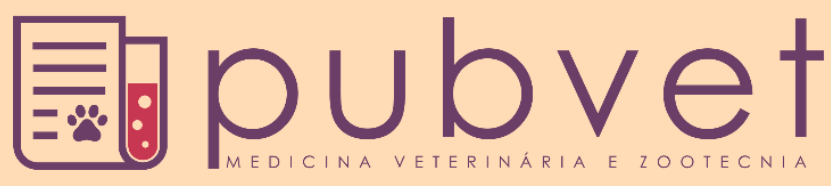

https://doi.org/10.31533/pubvet.v15n06a839.1-8

\title{
Grupo sanguíneo DEA-1 de cães e sua relação com reações transfusionais
}

\author{
Flávia Zandoná Puchalski ${ }^{1 *}$, Juliana Murasaki ${ }^{1}{ }^{\bullet}$, Pricilla Pozzatti ${ }^{2} \bullet$, Carlos Eduardo \\ Nogueira Martins ${ }^{3}$ ( (D)
}

${ }^{I}$ Discente de Medicina Veterinária do Instituto Federal Catarinense-Campus Araquari- Santa Catarina, Brasil.

${ }^{2}$ Professora e orientadora do Instituto Federal Catarinense-Campus Araquari, Departamento de Laboratório Clínico Veterinário. Araquari-SC, Brasil. ${ }^{3}$ Professor do Instituto Federal Catarinense- campus Araquari. Departamento de estatística e zootecnia. Araquari-SC,Brasil.

*Autor para correspondência, E-mail: flaviapuchalski@gmail.com

Resumo. O presente trabalho teve como propósito de testar as reações do sangue de cães de tipagem sanguínea indefinida com sangue de cães identificados como "Dog Erythorocyte Antigen" (DEA) 1 negativos, provenientes da cidade de Jaraguá do Sul, SC, Brasil. Para tanto, foram selecionados 12 cães sem raça definida (SRD) para compor 2 grupos: Grupo 1 com duas amostras de cães (A e B) DEA-1 negativos; Grupo 2: 10 cães de tipagem sanguínea desconhecida. A tipagem sanguínea dos cães do grupo 1 foi identificada através do teste RapidVet ${ }^{\circledR}$ que foi realizado em 5 cães, dos quais 2 DEA-1 negativos foram selecionados. Em seguida o teste de Reação Cruzada Maior, técnica rápida em lâmina de microscopia, foi realizado em todas as amostras do grupo 2 com as amostras do grupo 1. As lâminas foram analisadas em busca de aglutinação para definição de compatibilidade entre as amostras. Dos 5 cães que passaram pelos testes de tipagem sanguínea, 4 eram DEA-1 negativos e 1 DEA-1 positivo. Dos dez testes de reação cruzada realizados com a amostra do cão $\mathrm{A}$ do grupo $1,70 \%$ das amostras se mostraram compatíveis, ou seja, não apresentaram aglutinação micro ou macroscópica e $30 \%$ incompatíveis, apresentando aglutinação. Das reações cruzadas feitas com a amostra do cão B do grupo 1, 40\% foram classificadas como compatíveis e 60\% de amostras incompatíveis. A análise estatística com os resultados apresentados não demonstra diferença entre as frequências de resultados compatíveis e incompatíveis com as amostras dos cães A e B e, comparando os resultados das amostras, houve uma tendência $(0,09)$ de diferença na frequência dos resultados. O cálculo do risco de um cão DEA-1 negativo receber sangue de um cão DEA-1 positivo em uma primeira transfusão resultou em 24,75\% e de $6,15 \%$, do mesmo cão receber sangue incompatível em uma segunda transfusão. A frequência encontrada de animais com sangue DEA-1 negativo foi maior que a frequência de DEA-1 positivo. A baixa incidência do grupo DEA-1 demonstra uma necessidade de um estudo mais profundo e minucioso para que seja possível determinar a real incidência desse grupo sanguíneo no município de Jaraguá do Sul, SC. Diante dos resultados obtidos, conclui-se que a frequência encontrada de animais com sangue DEA-1 negativo foi maior que a frequência de DEA-1 positivo no município de Jaraguá do Sul, SC e o risco de incompatibilidade sanguínea durante uma possível primeira e segunda transfusões entre os cães desse estudo foram de $24,75 \%$ e $6,15 \%$ respectivamente.

Palavras-chave: Antígenos eritrocitários, reação cruzada, tipagem sanguínea, transfusão sanguínea

\section{Dogs blood group DEA-1 and its relation to transfusion reactions}

Abstract. The purpose of this study was to test the blood reactions between the blood of undefined blood-type dogs and the blood of negative "Dog Erythrocyte Antigen" (DEA) 1 dogs from Jaraguá do Sul-SC, Brazil. Therefore, 12 mongrel dogs were selected to form 2 
groups: Group 1 with two dog blood samples (A and B) negative DEA-1; Group 2: 10 dogs of undefined blood-type. The blood-type of the Group 1 dogs was identified by RapidVet ${ }^{\circledR}$ test that was performed in 5 dogs of which 2 negative DEA-1 were selected. Subsequently, the Major Crossmatch test, rapid method in microscope slide, was performed between all the Group 2 blood samples and both Group 1 samples. The slides were examined for macroscopic and microscopic agglutination to define the samples compatibility. From the 5 blood-type tested dogs, 4 were negative DEA- 1 and 1 was positive DEA-1. From the ten crossmatch tests performed with the group $1 \mathrm{dog}$ A sample, $70 \%$ of the samples were compatible, they did not present micro or macroscopic agglutination and $30 \%$ were incompatible, presented agglutination. From the crossmatch tests performed with the group 1 dog B sample, $40 \%$ were compatible and $60 \%$ of the samples were incompatible. The statistical analysis of the obtained results does not show difference between the frequencies of the compatible and incompatible results of the A and B dog samples and comparing the samples results there was a tendency (0.09) of difference in the results frequency. The risk calculation of a negative DEA-1 dog receiving blood of a positive DEA-1 dog at a firsttime transfusion was $24,75 \%$ and $6,15 \%$ of this same dog receiving incompatible blood in a second transfusion. The frequency of negative DEA-1 blood dogs was higher than the frequency of positive DEA-1 blood dogs. The low incidence of the DEA-1 group establishes a need of further and more detailed study to determine the real incidence of this blood group in Jaraguá do Sul, SC. Facing the results, it was concluded that the negative DEA-1 frequency was higher than the positive DEA-1 fequency in Jaraguá do Sul, SC and that the risk of blood incompatibility between the studied dogs during a first and second transfusions were $24,75 \%$ and $6,15 \%$, respectively.

Keywords: Erythrocyte antigens, crossmatch, blood typing, blood transfusion

\section{Grupo sanguíneo DEA-1 de los perros y su relación con las reacciones transfusionales}

Resumen. El presente trabajo objetivó probar las reacciones sanguíneas de perros de tipo sanguíneo indefinido con sangre de perros identificados como Dog Erythorocyte Antige DEA-1 negativos, de la ciudad de Jaraguá do Sul, SC, Brasil. Para ello, se seleccionaron 12 perros mestizos (SRD) y se dividieron en 2 grupos: Grupo 1 con dos muestras de perros (A y B) DEA-1 negativos; Grupo 2: 10 perros de tipo sanguíneo desconocido. La tipificación sanguínea de los perros del grupo 1 se identificó mediante la prueba RapidVet ${ }^{\circledR}$, que se realizó en 5 perros, de los cuales se eligieron 2 DEA-1 negativos. A continuación, se efectuó la prueba de reacción cruzada mayor, una técnica rápida de portaobjetos de microscopía, en todas las muestras del grupo 2 con las muestras del grupo 1. Se analizaron los portaobjetos para determinar la aglutinación y definir la compatibilidad entre las muestras. De los 5 perros que se sometieron a las pruebas de determinación del grupo sanguíneo, 4 dieron negativo en DEA-1 y 1 positivo en DEA-1. De las diez pruebas de reacción cruzada realizadas con la muestra del perro A grupo 1, el 70\% de las muestras fueron compatibles, es decir, no presentaron aglutinación micro o macroscópica y el $30 \%$ incompatibles, revelando aglutinación. De las reacciones cruzadas realizadas con la muestra de perro B del grupo 1, el $40 \%$ se clasificaron como compatibles y el $60 \%$ de muestras incompatibles. El análisis estadístico con los resultados presentados no muestra diferencia alguna entre las frecuencias de resultados compatibles e incompatibles con las muestras de los perros A y B y, comparando los resultados de las muestras, hubo una tendencia (0.09) de diferencia en la frecuencia de los resultados. El cálculo del riesgo de que un perro con DEA-1 negativo recibiera sangre de un perro con DEA-1 positivo en una primera transfusión proveyó como resultado que el $24,75 \%$ y el $6,15 \%$ del mismo perro recibieran sangre incompatible en una segunda transfusión. La frecuencia encontrada en animales con sangre DEA-1 negativa fue mayor que la frecuencia de DEA-1 positiva. La baja incidencia del grupo DEA-1 demuestra la necesidad de un estudio más profundo y detallado para que sea posible determinar la incidencia real de este grupo sanguíneo en el 
municipio de Jaraguá do Sul, SC. Se concluye que la frecuencia encontrada de animales con sangre DEA-1 negativa fue superior a la frecuencia de DEA-1 positiva en el municipio de Jaraguá do Sul, SC y el posible riesgo de incompatibilidad sanguínea durante las primeras y segundas transfusiones entre los perros de este estudio fueron del $24,75 \%$ y del $6,15 \%$, respectivamente.

Palabras-clave: Antígenos de eritrocitos, reacción cruzada, tipificación sanguínea, transfusión de sangre

\section{Introdução}

A transfusão sanguínea teve seus primeiros registros no século XVII e consiste em realizar a transferência do sangue e/ou seus componentes de um doador para um receptor, com objetivo de corrigir temporariamente o quadro clínico apresentado (González \& Silva, 2008; Núñez \& Bouda, 2007; Stockham \& Scott, 2011). Atualmente, os bancos de coleta podem obter diversos subprodutos do sangue, sendo cada um deles indicado para diferentes situações. A utilização de hemo-competentes específicos para o quadro do animal resulta na não exposição do cão aos antígenos estranhos desnecessários, diminuindo possíveis reações adversas (Nelson \& Couto, 2015; Reis \& Di Gregorio, 2017).

Os tipos sanguíneos dos cães são definidos pelo sistema "Dog Erythorocyte Antigen" (DEA), o qual baseia-se na presença de antígenos espécie-específicos na membrana dos eritrócitos. Esses antígenos quando transferidos para um animal não compatível são identificados como estranhos e geram respostas imunes como hemólise ou produção de anticorpos. Os anticorpos antieritrocitários podem ser decorrentes de uma sensibilização prévia por exposição aos antígenos, ou de ocorrência natural, sendo esta, em casos específicos, como a gestação em cadelas (Nelson \& Couto, 2015; Novais \& Magron, 2018; Stockham \& Scott, 2011).

A determinação do tipo sanguíneo dos cães previamente a transfusão sanguínea reduz as chances de ocorrência de reações adversas. Para verificar se o sangue pode ser transfundido, são realizados os testes de reação cruzada maior e menor (Stockham \& Scott, 2011). A não realização dos testes de triagem coloca em risco a vida do animal, visto que podem ocorrer reações hemolíticas graves, piorando o quadro prévio do paciente (González \& Silva, 2008; Núñez \& Bouda, 2007; Stockham \& Scott, 2011).

Diante da importância clínica e do aumento na frequência do uso de transfusões sanguíneas na rotina da clínica médica de pequenos animais, este trabalho tem como objetivo testar as reações do sangue de cães de tipagem sanguínea indefinida com sangue de cães DEA-1 negativo.

\section{Material e métodos}

Esse projeto possui aprovação no Comitê de Ética para Uso de Animais (CEUA) sob protocolo número 338/2020. Todos os cães participantes selecionados eram sem raça definida, de peso e idade variados, provenientes do município de Jaraguá do Sul, Santa Catarina. A seleção dos animais ocorreu de forma voluntária por seus proprietários.

Foram selecionados 15 cães divididos em dois grupos. No primeiro grupo foram escolhidos aleatoriamente, cinco cães nos quais foi realizado o teste de tipagem sanguínea RapidVet ${ }^{\circledR}$ de forma sequencial. Dos cinco, dois que apresentaram resultado DEA-1 negativo foram selecionados para compor o grupo 1 do experimento, simulando cães receptores no processo de transfusão sanguínea (Cão A e Cão B). Os outros 10 cães restantes, dos quais o DEA não era conhecido, integraram o grupo 2 do experimento, simulando os doadores.

Todas as coletas de sangue foram realizadas com agulha de calibre $22 \mathrm{~g}(0,70 \times 25)$ e seringa de 5 $\mathrm{mL}$, por meio de punção da veia jugular. A contenção física foi realizada visando o mínimo estresse dos cães e quando necessário, foi utilizado focinheira. As amostras sanguíneas foram armazenadas em tubos contendo EDTA e identificadas com o número do animal e grupo pertencente.

Todas as amostras do grupo 2 passaram pelo teste de Reação Cruzada Maior através da técnica rápida em lâmina de microscopia, tal como descrita no Manual of Canine and Feline Hematology and Transfusion Medicine (2012), com as duas amostras provenientes do grupo 1. Todas as amostras foram centrifugadas a uma velocidade de 1000-1500 g por 5-10 minutos, visando a separação do plasma. Após 
centrifugação, o plasma foi transferido para outro tubo de ensaio. Com o plasma separado, foi preparada uma solução a $4 \%$ do concentrado de eritrócitos com solução salina, sendo todas devidamente identificadas. Em seguida, para cada teste de reação cruzada realizado, foram montadas quatro lâminas de microscopia, cada uma identificada com: controle doador, controle receptor, Reação cruzada maior e Reação cruzada menor. Em cada lâmina eram misturadas, com o auxílio de uma pipeta limpa, uma gota de plasma com uma gota da solução de eritrócitos, sendo:

- Controle do doador: Eritrócitos doador + plasma doador;

- Controle receptor: Eritrócitos receptor + plasma receptor;

- Reação Cruzada Maior: Eritrócitos doador + plasma receptor;

- Reação Cruzada Menor: Eritrócitos receptor + plasma doador.

Essas lâminas foram analisadas em busca de aglutinação macroscópica e microscópica dentro de dois e cinco minutos, respectivamente. No microscópio, foram utilizadas as objetivas de 40x e 100x.

Os dados obtidos foram analisados pelo teste do Qui-quadrado com o auxílio do programa estatístico jamovi, versão 1.2, considerando o nível de significância de 5\%.

\section{Resultados e discussão}

A classificação sanguínea dos cães é dividida em sete grupos sanguíneos que compõem o sistema DEA, sendo eles: DEA-1 (com subgrupos 1.1, 1.2 e 1.3), DEA-3, DEA-4, DEA-5, DEA-6, DEA-7 e DEA-8 (Hohenhaus, 2004). De acordo com Pereira \& Reichmann (2011), os subgrupos sanguíneos DEA-1.1, 1.2 e o grupo DEA-7 possuem o maior potencial antigênico, sendo o grupo DEA-1 capaz de gerar fortes estimulações antigênicas para a produção de anticorpos em um receptor não compatível. Por isso, o grupo tem sido considerado de maior importância quando se refere ao procedimento de transfusões sanguíneas e foi o grupo escolhido para a realização da presente pesquisa.

Inicialmente, o grupo 1, seria composto por duas amostras de cães DEA-1 positivos, entretanto, dos 5 animais submetidos ao RapidVet ${ }^{\circledR}, 4$ amostras apresentaram resultado de DEA-1 negativo, conforme mostram as Figuras $1_{\mathrm{a}}, \underline{1_{\mathrm{b}}}, \underline{1}_{\mathrm{c}} \mathrm{e} \underline{1}_{\mathrm{d}}$. Apenas 1 amostra apresentou-se positivo a esse grupo sanguíneo, demonstrado na Figura 2. Optou-se então pela mudança na metodologia do projeto, utilizando os cães A e B, sendo ambos DEA-1 negativos, para simularem cães receptores durante uma transfusão sanguínea. Os resultados obtidos nos testes de tipagem sanguínea foram um achado não esperado e revelam uma maior incidência do grupo sanguíneo DEA-1 negativo, o que contrapõe os resultados encontrados por Ferreira et al.(2011), Novais et al. (1999), Novais et al. (2004), Novais et al. (2019) e Vizzoni \& Medeiros (2017). Entretanto, o encontro de uma maior porcentagem de DEA-1 negativos concorda com os trabalhos de Ejima et al. (1986) e Swisher \& Young (1961) que encontraram valores menores de frequência de DEA-1 quando trabalharam com cães mestiços. Essa divergência de resultados reforça a afirmação de Novais et al. (2019) e Souza et al. (2014) de que a frequência do grupo sanguíneo DEA-1 varia de forma significativa de acordo com a raça e localidade geográfica do animal, podendo ser um fator decorrente do município de Jaraguá do Sul, origem os cães participantes. Como todas as amostras sanguíneas eram provenientes de cães sem raça definida, a variação encontrada teve influência dos genes raciais de cada indivíduo, além disso a menor porcentagem de cães DEA-1 positivo pode ser decorrente de um fator local do município em questão, tendo em vista que todos os cães participantes eram provenientes de Jaraguá do Sul, Santa Catarina.

Dos dez testes de reação cruzada realizados com a amostra do cão A do grupo 1,70\% das amostras se mostraram compatíveis, ou seja, não apresentaram aglutinação micro ou macroscópica e 30\% incompatíveis, apresentando aglutinação. Das reações cruzadas feitas com a amostra do cão B do grupo 1, 40\% foram classificadas como compatíveis e $60 \%$ de amostras incompatíveis (Tabela 1). Por ambas as amostras do grupo 1 serem classificadas como DEA-1 negativas, observou-se que houve uma diferença na compatibilidade das amostras do grupo $2 \mathrm{com}$ as amostras do grupo 1. Uma possível explicação, seria o fato de cães poderem apresentar mais de um tipo de antígeno sanguíneo na membrana de seus eritrócitos, portanto, pertencerem a mais de um grupo sanguíneo concomitantemente (Hale, 1995; Lanevschi \& Wardrop, 2001; Novais \& Magron, 2018; Symons \& Bell, 1991). A aglutinação ocorrida na reação cruzada das amostras 1,3 e 8 com apenas uma das amostras do grupo 1 poderia ser 
decorrente da incompatibilidade sanguínea de outro grupo sanguíneo que não teria sido analisado nesse estudo (Tabela 2).

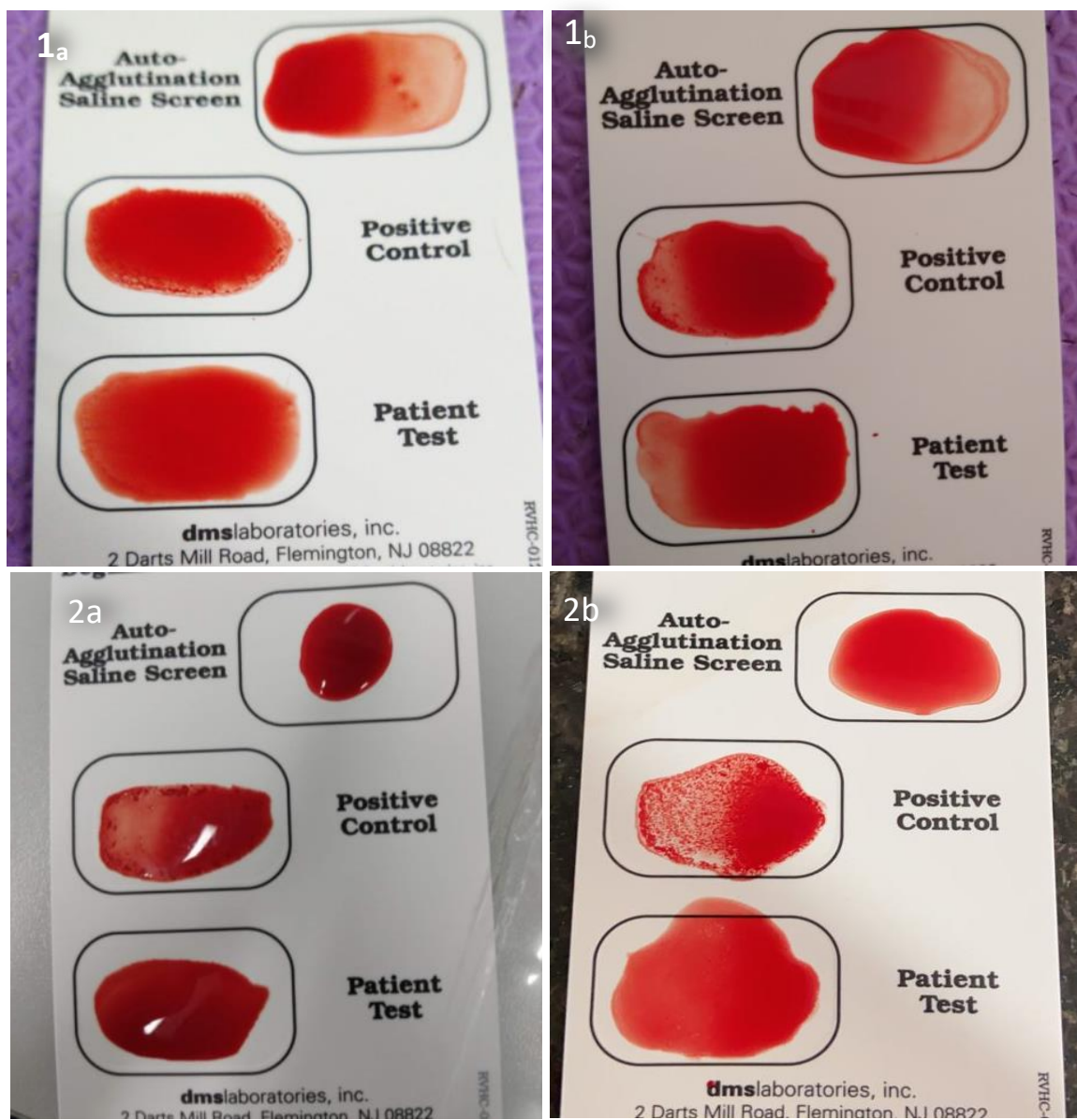

Figuras 1a e 1b. Teste de tipagem sanguínea Rapidvet $®$ dos cães A e B com resultado de DEA-1 negativo. Não há presença de aglutinação em nenhum dos poços "Patient Test".

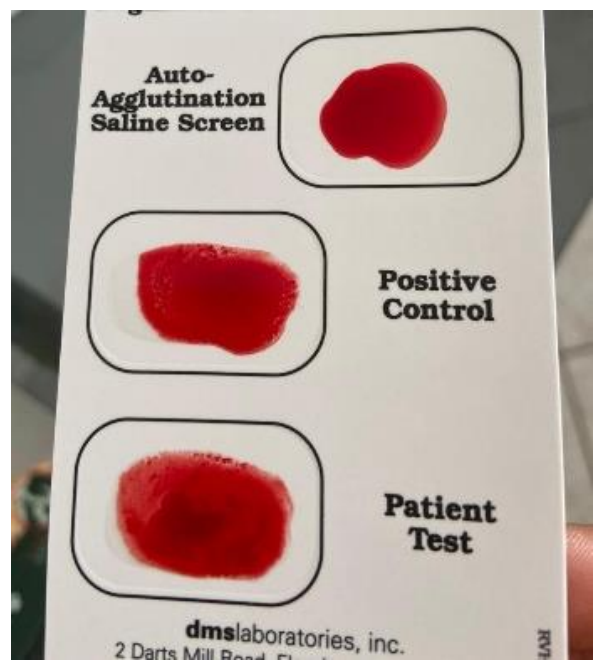

Figura 2. Teste de tipagem sanguínea Rapidvet ${ }^{\circledR}$ do cão E com resultado de DEA-1 positivo. Há presença de aglutinação no poço "Patient Test".

Tabela 1. \% de amostras compatíveis do grupo 2 nos respectivos testes com amostras dos cães A e B do grupo 1.

\begin{tabular}{lcccc}
\hline Amostra & \multicolumn{4}{c}{ Compatibilidade das amostras do grupo 2 com a amostras do grupo 1 } \\
\cline { 2 - 5 } Grupo 1 & Controle doador & Reação cruzada maior & Reação cruzada menor & Controle receptor \\
\hline Cão A & $100 \%$ & $70 \%$ & $70 \%$ & $100 \%$ \\
Cão B & $100 \%$ & $40 \%$ & $40 \%$ & $100 \%$ \\
\hline
\end{tabular}


Tabela 2. Classificação da compatibilidade das amostras do grupo 2 nos testes submetidas com as amostras do grupo 1.

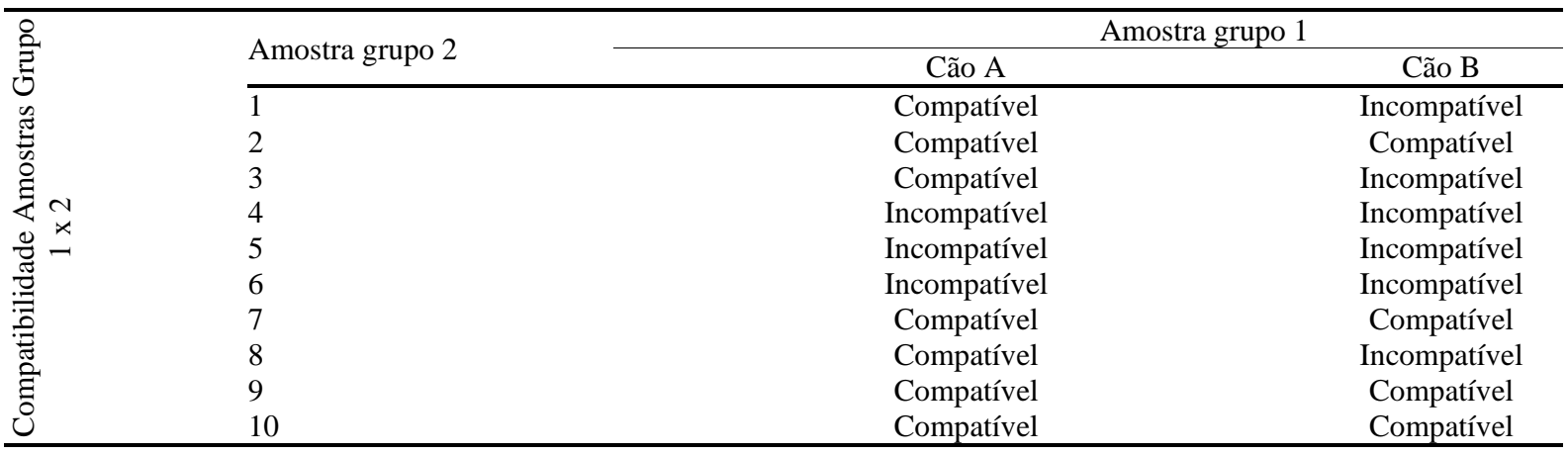

No presente trabalho, a determinação de compatibilidade ou incompatibilidade entre as amostras baseou-se na realização e interpretação da reação de aglutinação em lâmina descrita no Manual of Canine and Feline Hematology and Transfusion Medicine (2012). A Figura 3 demonstra a aglutinação ocorrida no teste de reação cruzada, com resultado de incompatibilidade sanguínea entre as amostras. As formações encontradas no exame microscópico se assemelham àquelas descritas pelos outros estudos encontrados. Ainda de acordo com o Manual of Canine and Feline Hematology and Transfusion Medicine (2012), alguns cães podem possuir níveis muitos baixos de aloanticorpos, podendo não ser detectados no teste de reação cruzada. Desse modo, torna-se fundamental a monitoração constante do paciente durante todo o procedimento para que seja possível a detecção precoce de qualquer sinal de reação transfusional e prontamente instituir o tratamento necessário (Maia et al., 2019; Morikawa et al., 2010).

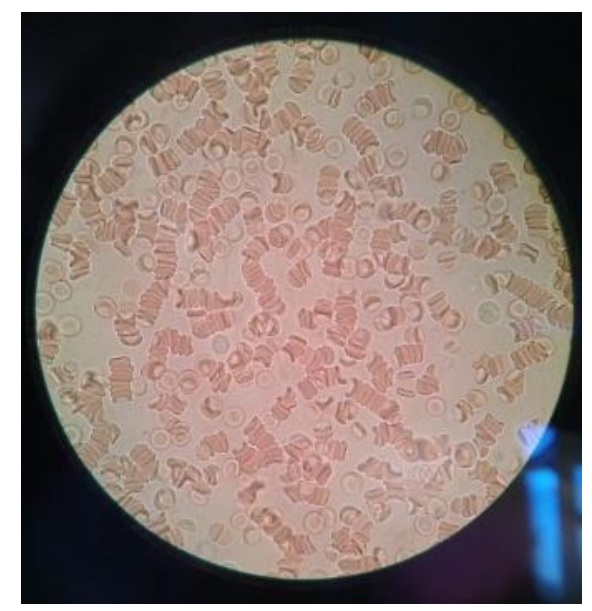

Figura 3. Incompatibilidade sanguínea entre amostras demonstrada pela aglutinação intensa entre os eritrócitos. Aumento de 100x.

Tabela 3. Teste de contingência de compatibilidade das amostras do grupo 2 nos testes submetidas com as amostras do grupo 1.

\begin{tabular}{|c|c|c|c|c|c|}
\hline \multirow{2}{*}{ Cão A } & \multicolumn{2}{|c|}{ Cão B } & \multirow{2}{*}{ Total** } & \multirow{2}{*}{$X^{2}$} & \multirow{2}{*}{$\mathrm{p}$} \\
\hline & Compatível & Incompatível & & & \\
\hline$\stackrel{0}{\Xi}$ Compatível & 4 & 3 & 7 & & \\
\hline 㫯 Incompatível & 0 & 3 & 3 & 2,86 & 0,091 \\
\hline \& Total* & 4 & 6 & 10 & & \\
\hline
\end{tabular}

Segundo Lanevschi \& Wardrop (2001) e Pereira \& Reichmann (2011), as reações que podem ocorrer com a transfusão de um sangue incompatível variam de reações imediatas (ocorrem algumas horas após a transfusão) a reações tardias (podendo ocorrer dias após o procedimento). Essa variação é diretamente relacionada aos tipos de antígenos incompatíveis envolvidos. As reações de incompatibilidade decorrentes do antígeno do grupo sanguíneo DEA-1 são caracterizadas como agudas, intravasculares e graves. Em contrapartida, as reações mediadas por anticorpos contra os grupos sanguíneos DEA-3, 5 e 7 são caracterizadas por sinais clínicos de menor gravidade, além de sequestro esplênico com hemólise 
extravascular, resultando em um menor tempo de vida dos eritrócitos transfundidos (Novais et al., 2004; Souza et al., 2014). Diante disso, é conferida uma maior importância ao grupo sanguíneo DEA-1, mesmo que a ocorrência de antígenos naturais para esse tipo sanguíneo seja extremamente rara, e que seja necessária uma sensibilização prévia, como discutido por Lanevschi \& Wardrop (2001) e Souza et al., (2014).

\section{Conclusão}

A frequência encontrada de animais com sangue DEA-1 negativo foi maior que a frequência de DEA1 positivo no município de Jaraguá do Sul, SC. O risco de incompatibilidade sanguínea durante uma possível primeira e segunda transfusão entre os cães desse estudo foram de $24,75 \%$ e 6,15\% respectivamente.

\section{Agradecimentos}

Aos laboratórios Badanievet e Vetex por possibilitarem a execução deste estudo científico.

\section{Referências}

Day, M. J \& Kohn, B. (2012). Manual of Canine and Feline Hematology and Transfusion Medicine. British Small Animal Veterinary, 2nd Edition, 320p.

Ejima, H., Kurokawa, K., \& Ikemoto, S. (1986). Phenotype and gene frequencies of red blood cell groups in dogs of various breeds reared in Japan. The Japanese Journal of Veterinary Science, 48(2), 363-368. https://doi.org/10.1292/jvms1939.48.363.

Ferreira, R. R. F., Gopegui, R. R., \& Matos, A. J. F. (2011). Frequency of dog erythrocyte antigen 1.1 expression in dogs from Portugal. Veterinary Clinical Pathology, 40(2), 198-201. https://doi.org/10.1111/j.1939-165X.2011.00311.x.

González, F. H. D., \& Silva, S. C. (2008). Patologia clínica veterinária: texto introdutório. Universidade Federal do Rio Grande do Sul.

Hale, A. S. (1995). Canine blood groups and their importance in veterinary transfusion medicine. Veterinary Clinics: Small Animal Practice, 25(6), 1323-1332. https://doi.org/10.1016/S01955616(95)50157-3.

Hohenhaus, A. E. (2004). Importance of blood groups and blood group antibodies in companion animals. Transfusion Medicine Reviews, 18(2), 117-126. https://doi.org/10.1016/j.tmrv.2003.12.003.

Lanevschi, A., \& Wardrop, K. J. (2001). Principles of transfusion medicine in small animals. The Canadian Veterinary Journal, 42(6), 447-454.

Maia, J., Sousa, A. V., \& Martins, R. R. (2019). Importância dos cuidados de enfermagem na transfusão de cães e gatos. Ciência Veterinária UniFil, 1(3), 66-68. https://doi.org/.

Morikawa, M. K., Bochio, M. M., Pincelli, V. A., Freire, R. L., \& Pereira, P. M. (2010). Monitoração e avaliação clínica da eficácia da transfusão de sangue total e concentrado de hemácias em cães. Pesquisa Veterinária Brasileira, 30(8), 665-669. https://doi.org/10.1590/S0100736X2010000800010.

Nelson, R. W., \& Couto, C. G. (2015). Medicina interna de pequenos animais (Issue 1). Elsevier Editora.

Novais, A A, Fagliari, J. J., \& Santana, A. E. (2004). Prevalência dos antıgenos eritrocitários caninos (DEA-dog erythrocyte antigen) em caes domésticos (Canis familiaris) craidos no Brasil.[DEA prevalence in domestic dogs (Canis familiaris) reared in Brazil. Ars Veterinaria, 20(2), 212-218. https://doi.org/10.1590/S1413-95961999000100004.

Novais, A A, \& Magron, H. F. (2018). Canine blood groups: a review. Scientific Electronic Archives, 11(5), 183-187. https://doi.org/10.36560/1152018670.

Novais, Adriana Alonso, Magron, H. F., Kataoka, A., Canei, D. H., Silva, J. P., \& Vasconcelos, A. L. (2019). Frequência do grupo sanguíneo DEA 1.1 em cães atendidos no Hospital Veterinário da UFMT (Sinop/MT), risco de sensibilização de cães DEA 1 negativos e da ocorrência de reação transfusional hemolítica por ocasião de uma segunda transfusão de sangue. Revista Brasileira de 
Ciência Veterinária, 26(2), 51-54. https://doi.org/10.4322/rbcv.2019.010.

Novais, Adriana Alonso, Santana, A. E., \& Vicentin, L. A. (1999). Prevalence of DEA 1 canine blood group system in dogs (Canis familiaris, Linnaeus, 1758) reared in Brazil. Brazilian Journal of Veterinary Research and Animal Science, 36(1). https://doi.org/10.1590/S141395961999000100004.

Núñez, O. L., \& Bouda, J. (2007). Patología Clínica Veterinaria: Vol. 1. ed.

Pereira, P. M., \& Reichmann, P. (2011). Tranfusão de sangue e seus derivados. In S. F. Andrade (Ed.), Manual de terapêutica veterinária (pp. 579-591). Roca, Brasil.

Reis, N. M., \& Di Gregorio, M. C. (2017). Tipos sanguíneos em cães domésticos (Canis familiaris): revisão de literatura. Revista de Ciência Veterinária e Saúde Pública, 4, 147. https://doi.org/10.4025/revcivet.v4i0.39830.

Souza, S. L., Stopiglia, A. J., Gomes, S. G. R., Ulata, S. K., Moroz, L. R., \& Fantoni, D. T. (2014). Estudo da frequência dos antígenos eritrocitários caninos $1,1.1$ e 7 e risco de transfusão incompatível em cães de diferentes raças e mestiços da região metropolitana da cidade de São Paulo-SP, Brasil. Brazilian Journal of Veterinary Research and Animal Science, 51(4), 316-323. https://doi.org/10.11606/issn.1678-4456.v51i4p316-323.

Stockham, S. L., \& Scott, M. A. (2011). Fundamentos de patologia clínica veterinária. In Guanabara Koogan (Vol. 8).

Swisher, S. N., \& Young, L. E. (1961). The blood grouping systems of dogs. Physiological Reviews, 41(3), 495-520. https://doi.org/10.1152/physrev.1961.41.3.495.

Symons, M., \& Bell, K. (1991). Expansion of the canine A blood group system. Animal Genetics, 22(3), 227-235. https://doi.org/10.1111/j.1365-2052.1991.tb00672.x.

Vizzoni, A. G., \& Medeiros, M. Â. S. (2017). Imunohematologia veterinária: antígenos eritrocitários caninos. Arquivos de Ciências Veterinárias e Zoologia Da UNIPAR, 20(4), 241-246. https://doi.org/10.25110/arqvet.v20i4.2017.5682.

Histórico do artigo:

Recebido: 12 de dezembro de 2020.

Aprovado: 4 de janeiro de 2021.
Licenciamento: Este artigoé publicado na modalidade Acesso Aberto sob a licença Creative Commons Atribuição 4.0 (CC-BY 4.0), a qual permite uso irrestrito, distribuição, reprodução em qualquer meio, desde que o autor e a fonte sejam devidamente creditados. 